\title{
Connaître la FMH pour savoir s'en servir
}

\author{
Anne-Geneviève Bütikofera, Jacqueline Wettstein ${ }^{b}$ \\ a Secrétaire générale de la $\mathrm{FMH} ;{ }^{b}$ Cheffe de la division Communication de la FMH
}

\begin{abstract}
Que fait concrètement l'organisation professionnelle des médecins? Pour répondre à cette question qui revient souvent, la FMH a invité les médecins nouvellement engagés en politique professionnelle et les secrétaires généraux des organisations médicales à un après-midi d'information leur offrant ainsi l'occasion de se familiariser avec les activités de la FMH et de discuter des thèmes actuels de la politique de la santé.
\end{abstract}

Reprendre la présidence d'une société de discipline, entrer au comité d'une organisation faîtière ou diriger le secrétariat général d'une société cantonale sont autant de missions aussi passionnantes que diversifiées. Pour présenter la FMH, ses fonctionnements et ses activités, la secrétaire générale de la FMH a pour la deuxième fois invité les délégués nouvellement élus à un programme d'introduction. Les présentations et les ateliers ont été l'occasion de recueillir des informations et d'échanger sur les thèmes actuels de la politique de santé.

\section{Pour l'indépendance de la profession libérale de médecin}

Avec plus de 70 organisations médicales et près de 40000 membres, la FMH est aujourd'hui l'une des organisations professionnelles les plus importantes de Suisse et fait partie des principaux acteurs du domaine de la santé. La première association des médecins suisses a vu le jour à l'époque de la Révolution fran-

La première association des médecins suisses a vu le jour à l'époque de la Révolution française.

çaise, suivie au $18^{\mathrm{e}}$ siècle par la création de différentes organisations régionales qui se sont progressivement réunies en organisations intercantonales. La pierre fondatrice de la FMH a été définitivement posée en 1901 et les sociétés de discipline sont apparues à partir de 1930. Toutes ces organisations défendent une valeur commune: les médecins doivent pouvoir exercer leur profession librement, de manière indépendante, autonome et responsable.
En tant qu'organisation faîtière du corps médical suisse, la FMH représente les intérêts de ses membres face aux autorités et autres institutions mais aussi visà-vis du grand public. Plusieurs organes au sein de la

Les nombreux ateliers ont permis aux participants de se familiariser avec les différentes prestations de la FMH.

FMH permettent une répartition équilibrée de la défense des intérêts des membres: la Chambre médicale, le parlement des médecins, fixe les grandes lignes de la politique de la FMH; l'Assemblée des délégués traite toutes les questions essentielles de la politique de la santé et de politique professionnelle; le Comité central représente la FMH à l'égard de l'opinion publique et des autorités; et enfin la votation générale, organe suprême qui permet d'exercer la démocratie directe auprès de tous les membres ayant le droit de vote, de manière analogue à la votation populaire.

\section{Gouverner, c'est prévoir}

A l'image de l'adage cité par le président de la FMH "Gouverner, c'est prévoir», la FMH a toujours regardé de l'avant et c'est ce qu'elle a fait lorsqu'elle a rédigé le Code de déontologie. Ce dernier définit pour ses membres les devoirs professionnels les plus importants et diverses règles éthiques, comme la manière de traiter dignement les patients en fin de vie ou la collaboration entre le corps médical et l'industrie. Le Code de déontologie établit ainsi une sorte de label qualité. La FMH a également agi de manière adéquate lorsque, suite à l'entrée en vigueur des Accords bilatéraux il y a 
10 ans, il n'était plus certain que ce serait elle qui continuerait d'organiser la formation postgraduée et continue. En 2009, elle a donc créé l'Institut suisse pour la formation médicale postgraduée et continue (ISFM). En tant qu'organe autonome de la FMH, l'ISFM conseille et gère plus de 10000 médecins-assistants en formation et s'occupe de la certification de près de 1500 établissements de formation postgraduée. L'ISFM s'est donc également présenté lors de cette occasion.

\section{De nombreux projets, des services pratiques et utiles}

Les nombreux ateliers ont permis aux participants de se familiariser avec les différentes prestations de la FMH et de s'informer des dossiers et travaux menés actuellement par la FMH. Le projet TARCO (TARMED Consensus) par exemple vise à trouver un dénominateur commun entre toutes les organisations représentées à la Chambre médicale afin de pouvoir poursuivre la révision du tarif ambulatoire. En plus de participer au développement continu de SwissDRG, la FMH travaille également à la mise sur pied de nouveaux tarifs hospitaliers comme ST Reha, le système tarifaire de la réadaptation hospitalière, et TARPSY, le système tarifaire de la psychiatrie hospitalière. Grâce à son travail de lobbying, la FMH a notamment obtenu que les forfaits par cas de TARPSY soient remplacés par des forfaits journaliers.

Correspondance: Fédération des médecins suisses (FMH) Brigitte Stöckli Elfenstrasse 18 Case postale 300 CH-3000 Berne 15 Tél. 0313591111 direction[at]fmh.ch sont les objectifs de la charte qualité de l'ASQM et comment la FMH participe au groupe de travail interprofessionnel GTI DEP pour concevoir un dossier du patient qui réponde aux besoins des médecins et des patients. Les différents stands d'information ont été un lieu d'échange offrant la possibilité de découvrir les offres telles que le guide juridique pour médecins, les recommandations de la FMH en matière de politique de la santé, le registre des médecins en ligne doctorfmh.ch, ou encore la Commission de déontologie, le Bureau d'expertises extrajudiciaires de la FMH et le profil professionnel des assistantes médicales.

\section{Séance plénière sur les points chauds de l'actualité}

L'après-midi d'information s'est achevé par une séance plénière consacrée aux thèmes choisis par les participants. Ces derniers en ont profité pour s'informer de l'avancement de la révision du tarif ambulatoire et de la mise en œuvre de la loi fédérale sur le dossier élec-

L'après-midi d'information s'est achevé par une séance plénière consacrée aux thèmes choisis par les participants.

tronique du patient. L'assemblée a été particulièrement critique en ce qui concerne le projet MARS de la Confédération, qui, selon les dispositions légales, oblige les cabinets médicaux et les centres ambulatoires à fournir leurs données. La FMH va poursuivre son engagement pour aider et soutenir le mieux possible ses membres et pour réduire les tâches administratives que le relevé implique. S’agissant de la consultation du règlement de traitement pour le projet MARS, la FMH est intervenue jusqu'ici avec succès et elle a ainsi obtenu une importante prolongation de délai.
Nous nous réjouissons de vous accueillir nombreux lors de la prochaine manifestation. 